\title{
La noción de «espíritu» en la filosofía de Wilhelm Dilthey
}

\author{
The Notion of «Spirit» in the Philosophy of \\ Wilhelm Dilthey \\ LUIS MARÍA LORENZO \\ CONICET (Argentina)
}

Recibido: 30/09/2015 Aceptado:14/10/2015

\section{RESUMEN}

La noción de espíritu es central en la filosofía de Dilthey. No es un concepto solamente utilizado para delimitar un campo de estudio científico, es sobre todo el resultado de la actividad del hombre en tanto ser histórico. No posee carga metafísica pues el espíritu es fruto del modo en que el presente, pasado y futuro se articula dentro de un curso vital. En el presente artículo se expondrá la noción de espíritu en Dilthey y su vínculo con la vida.

\section{PALABRAS CLAVE}

ESPÍRITU, VIDA, ACCIÓN, HISTORIA

\section{ABSTRACT}

The notion of 'spirit' is central to Dilthey's philosophy. It's not only a concept used to define a scientific field, but is mainly the result of the activity of human beings as historical beings. Likewise, it has not a metaphysical burden, because the spirit is fruit of the way in which present, past and future are articulated within the process of life. This article explains Dilthey's notion of spirit and its links to life.

\section{KEYWORDS}

SPIRIT, LIFE, ACTION, HISTORY

\section{INTRODUCCIÓN}

Wilhelm Dilthey desDe los Comienzos de su investigación filosófica 
encamina sus estudios en pos de encontrar una fundamentación para las ciencias del espíritu con la intención de, por un lado, brindarle un sustento científico metodológico y, por el otro, independizarla del dominio o subordinación de las ciencias naturales. Pero previamente a dicha operación sostiene que toda ciencia o toda teoría de la ciencia necesitan de un análisis gnoseológico que cimiente las bases de sus conocimientos. Para comprender este vasto proyecto es importante contar previamente con una aproximación a la noción de «espíritu» por él utilizada. Este concepto es frecuentemente usado por dicho autor en todas sus investigaciones, hecho que justifica un estudio sobre el mismo. En este sentido, el presente artículo no pretende realizar una exposición detallada de la obra de Dilthey, la cual es sumamente difícil de abordar por la forma «arbórea» en que escribe sus textos y articula sus conceptos, como también por su disposición esquiva a efectuar definiciones. Aquí el objeto de estudio es su noción de espíritu, la cual para ser comprendida requiere se efectúe un recorrido panorámico sobre su filosofía y las discusiones que operan de fondo. Para cumplir con dicho propósito en primer lugar se estudiará el contexto del surgimiento del proyecto diltheyano, la disputa entre ciencias naturales y ciencias del espíritu. En un segundo momento se presentará una breve reseña del concepto espíritu en la filosofía alemana y particularmente en Hegel pues Dilthey reconoce su influencia. Finalmente se expondrá la noción de espíritu en el propio Dilthey.

\section{CienCiAS DEL ESPÍRITU Y CIENCIAS NATURALES}

Dilthey utiliza la expresión «ciencias del espíritu» pues sostiene que el término «espíritu» es lo suficientemente impreciso para delimitar el vasto y diverso campo de estudio abarcado por dicha ciencia; otras formas (como ciencias morales) son en términos comparativos estrechas y restrictivas a la hora de dar cuenta de dichas multiplicidades y extensiones [ $c f$., Dilthey 1949, p. 14 (GS I, 5)]. ${ }^{1}$ Asimismo, reconoce que esta expresión pertenece a John Stuart Mills. No obstante, el término «ciencias del espíritu» no pertenece a la pluma de este último, el mismo es fruto de J. Schiel quien en 1849 tradujo al alemán su Sistema de la Lógica; allí propuso ciencias del espíritu como la traducción de «ciencias morales». ${ }^{2}$ Por otra parte en relación al origen de dicha expresión

1 Se utilizará el sistema de citas simplificado haciendo referencia en primera instancia a la traducción castellana y luego a su correspondencia con el original. En el caso de Dilthey se usará «GS» para hacer referencia al original seguido del volumen en números romanos. Se utilizará un formato similar para todo los casos de citas con referencia doble (traducción-original).

$2 C f$., Ritter, J. 1974, pp. 211ss.; Makkreel, R. 2010, p. 254; Makkreel, R. 1992, p. 36; cf., Apel, K-O. 1979, p. 17. Es importante remarcar que el concepto de «ciencias del espíritu» en Dilthey no es equivalente al de «ciencias morales» tal como las usa Mills. [cf. Ibid, pp. 35ss.]. 
Ritter sostiene que la aparición del término Geisteswissenschaften es muy poco clara. Subraya que tal vez la primera utilización se la puede encontrar en un escrito anónimo de 1787. Asimismo, se puede apreciar que la expresión «ciencias del espíritu» aparece en algunos escritos diltheyanos anteriores a la Introducción a las Ciencias del Espíritu. ${ }^{3}$ También la utiliza Hegel en la Fenomenología del Espíritu bajo la forma genitiva «Wissenschaft des Geistes» [cf., Hegel, G.W.F. 2006, p. 60 (Werk 3, 81)] y Dilthey hace lo propio en 1867 cuando reconoce que el problema de su generación es dilucidar la posibilidad de las Wissenschaften des Geistes [cf., Dilthey 1945, p. 345 (GS V, 13)].

En el contexto histórico donde Dilthey propone dicha denominación se puede observar dos modelos epistemológicos claramente contrapuestos. Por una parte, se encontraba la visión positivista que postulaba la existencia de un único método científico válido para todas las ciencias. Así, la sociología positivista toma el método de las ciencias naturales y sostiene que con él se puede «explicar» los hechos del mundo humano dándole seguridad a dichos saberes. En contraposición, surgen, principalmente en Alemania, corrientes filosóficas contrapuestas. Windelband y Ricker, enrolados en la escuela de Baden, proponen una visión dualista de la ciencia y dividen ciencias naturales y ciencias de la cultura. Dilthey, como heredero de esta «vuelta a Kant», comparte la visión dualista pero se distancia de estos autores por las implicancias universalistas y sesgadas asociadas a la idea de los «valores universales» como criterio que otorgara claridad, distinción y seguridad a los saberes relativos al mundo humano. También se opone al positivismo pues, según él, someten el mundo espiritual al conocimiento natural subordinando la psicología a la fisiología [cf., Dilthey 1978a, p. 393 (GS V, 53)]. Esto conlleva a dos supuestos lógicos que Dilthey considera indemostrables, uno la condicionalidad físico-material de la vida psíquica espiritual y el otro la imposibilidad del estudio de la experiencia interna ya que la misma es negada, todo es resumido a implicaciones lógicas y regularidades mutilando la realidad histórica [Dilthey 1978a, p. 134 (GS V, 92) / Dilthey 1949 p. 5 (GS I, p. xii)]. Así, para esta línea positivista de investigación las ciencias empíricas se limitarían, por tanto, a la búsqueda de uniformidades y sucesiones en los fenómenos dados en la «experiencia externa» ya que por «todas las partes del mundo se enlazan en la misma regularidad», «todo era racional y natural.» [Dilthey 1978b, pp. 134-135 (GS VIII, 104-5)]. Dilthey sostiene que el modelo positivista subsume el mundo espiritual a las leyes del mundo natural al considerar que los saberes sobre el mundo humano son dependientes de los adquiridos por las ciencias biológicas y las uniformidades por ellas detectadas

3 Esto se aprecia en los manuscritos de 1871 (cf., GS XVIII, 35) y de 1876 (cf., GS XVIII, 79) 
[(cf., Dilthey 1949, p. 107 (GS I, 105)]. De este modo, con su abordaje de la historia Comte desarrolla una filosofía naturalista de la historia [cf. Ibid., p. 106 (GS I, 104)].

Estas limitaciones conducen a Dilthey, dentro del análisis de las teorías de la ciencia, hacia una indagación sobre los mecanismos lógicos de investigación propios de las ciencias naturales y de las ciencias del espíritu. En lo que refiere al problema lógico, en el campo empírico, para él, tanto las ciencias naturales como las del espíritu presentan las mismas operaciones lógicas: inducción, análisis, construcción y comparación. La inducción es un saber de los datos sensibles que en el campo de las ciencias del espíritu aborda la "conexión estructural» de una «vida psíquica», su conexión real con otras unidades de vida y el mundo histórico y no como una abstracción ni como un fenómeno natural. Dilthey no desconoce que las ciencias naturales también analizan la conexión de estas manifestaciones sensibles pero, a su entender, lo hacen desde las representaciones y abstracciones cuantitativas, modos propios del conocimiento natural que aborda la «experiencia externa».

Dentro de la visión positivista los hechos espirituales solo pueden ser captados en tanto fenómenos naturales, a partir de la experiencia externa, negando la existencia de una experiencia interna. Dilthey entiende que, por un lado, se encuentran las ciencias naturales que estudian la vida desde su aspecto natural, a partir de la "experiencia externa»y, por el otro, las ciencias del espíritu que hacen lo propio al abordar la vida espiritual, a partir de la «experiencia interna». La diferencia entre experiencia interna y externa no debe aquí ser tomada literalmente sino más bien metafóricamente, como un recurso que busca remarcar la existencia de modos distintos de relacionarse con el mundo. La experiencia externa apunta a mostrar ese vínculo derivado o de segunda instancia que tiene el hombre con los objetos del mundo natural. Por su parte, la experiencia interna refiere a la relación íntima que el hombre tiene con el curso vital humano. El hombre se encuentra dentro de la temporalidad histórica de la cual se percata a partir de la vivencia, relaciones vivibles captadas por la experiencia interna [cf., Dilthey 1944a, p. 216 (GS VII, 192)]. Para Dilthey, la vida espiritual, como etapa más alta de la evolución de la tierra, es solo abarcable desde la vivencia propia de la vida psíquica. «La vida espiritual aparece en el suelo de lo físico; representa la etapa más alta de la evolución en la tierra.» [Ibid., p. 220 (GS VII, p. 196)]

El mundo humano se articula alrededor de las vivencias y sus exteriorizaciones, por ello, el modelo naturalista no puede captar correctamente las particularidades del curso histórico. Es importante señalar que si bien para Dilthey las ciencias del hombre tienen su campo propio de acción que no puede ser subordinado a los descubrimientos de las ciencias naturales esto no implica que queden totalmente separadas y no entren en contacto. La base 
de las ciencias del espíritu es la naturaleza pues allí el hombre realiza sus acciones y acuña sus fines y valores; pero sobre esta base la actividad humana hace emerger una realidad totalmente nueva, de la cual se ocupan las ciencias del espíritu [Ibid., pp. 140-1 (GS VII, 119)]. Esto permite apreciar que Dilthey reconoce puntos de contacto entre las ciencias de la naturaleza y las ciencias del espíritu. Ambas abordan la vida, una el aspecto físico (vida natural), la otra la forma psíquica y sus objetivaciones (vida espiritual). Desde la experiencia externa se captan los fenómenos naturales, se descubre sus leyes y se explican sus características; desde la experiencia interna los fenómenos propiamente humanos a los efectos de dar cuenta de las acciones y objetivaciones de la vida histórica. Que las ciencias del espíritu aborden la «experiencia interna» implica que ellas dan cuenta de las «conexiones estructurales» de las vidas psíquicas, «acciones objetivadas», especialmente aquellas expresadas en forma verbal o lingüística. Por ello, «En el mundo histórico no existe ninguna causalidad científico-natural porque causa, en el sentido de esta causalidad, implica que provoque efectos necesariamente, con arreglos a leyes; la historia sabe únicamente de relaciones de hacer y padecer, de acción y reacción.» [Ibid., p. 221 (GS VII, p. 197)].

El descubrimiento de estos postulados sobre la voluntad y la acción conducen a Dilthey a superar el mero análisis epistemológico ${ }^{4}$ para pasar a una indagación más amplia. Estas acciones de la voluntad expresan la conexión de vida de acuerdo con valores, significados y fines que se articulan históricamente en cambios que expresan el juego libre de energías (fuerzas, intenciones y elecciones) puesta en una o varias direcciones, las cuales no pueden ser comprendidas en los términos causales propuestos por las ciencias naturales.

En conexión con las categorías del hacer [acción] y padecer [reacción] tenemos la de fuerza. [...] La «fuerza» es en las ciencias naturales un concepto hipotético. Si se capta su validez, queda determinado por el principio de causalidad. En las ciencias del espíritu es la expresión categorial de algo vivible. Surge cuando nos encarnamos con el futuro, lo cual ocurre de los modos más diversos. [Ibid., pp. 226-7 (GS VII, p. 202)].

Así, Dilthey pasa de la teoría de la ciencia a la filosofía de la vida. Se puede decir, en términos generales, que la «vida» es para Dilthey un fenómeno omnipresente que se opone al racionalismo y a todas las filosofías del mero entendimiento. Si la vida es fuerza ella expresa tensiones, caminos y posibilidades que generaran distintas conexiones y estructuras. Esta fuerza

4 En este contexto se entiende por «epistemología» a la base teórica que busca la fundamentación de las ciencias del espíritu. 
vital es el curso sobre el cual se articula la acción humana y da forma al mundo histórico [Dilthey 1978a, p. 362 (GS VI, p. 313)]. La dimensión estructural de la vida psíquica, su forma adquirida, enuncia la propia dinámica estructural de la vida. Ella aparece como un telón de fondo en todas las producciones humanas, de ahí su importancia. Brevemente, se puede decir que la vida tiene un carácter holístico, expresa el curso temporal en el que se encuentra inmerso el hombre; sin embargo ella no tiene connotaciones de tipo metafísico ni cientificista, para Dilthey ella es facticidad, un curso, no una entidad constituida previamente a su manifestación. En otras palabras, la vida es un curso histórico-temporal que se presenta a la conciencia en conexiones estructurales vividas, expresa la dinámica de la acción humana en tanto flujo y conexiones fáctico-teleológicas (Rodi 2003, pp. 39-49). En el tiempo concreto (facticidad que articula presente-pasado-futuro) se establecen las conexiones históricas que hace posible la comprensión. En el curso vital se dan las formas culturales e históricas que se estructuran alrededor de la comunidad, generación y espíritu objetivo, formas estables de conexiones de valores, significados y fines históricos. El mundo humano es resultante de las acciones de los individuos dadas dentro de conexiones estructurales, tramas que moldea su identidad [Dilthey 1978a, pp. 362-3 (GS VI, p. 314)].

En pocas palabras, la vida es el fondo sobre el que actúan las unidades psíquicas de la vida (el hombre) quienes constituyen el mundo histórico; a su vez, es en el contacto con esta dimensión objetiva de la esfera cultural-socialhistórica donde cada una de las unidades de vida adquieren su estructura psíquica pues dentro de ella establecen conexiones diversas según distintos modos de entrelazar sus acciones. La «filosofía de la vida» propuesta por Dilthey es «filosofía de la acción», una indagación fáctica sobre el modo en que se generan históricamente los nexos efectivos (entramados de valores y fines). Es importante señalar que él reconoce tanto una dimensión natural como espiritual de la vida, no obstante solo se preocupará de la segunda. "Vida», como objeto de análisis de las ciencias del espíritu, es vida humana que gesta el mundo del espíritu. «Empleo la expresión 'vida' en las ciencias del espíritu limitándola al mundo humano.» [Ibid., p. 362 (GS VI, p. 314)]. La vida se presenta al hombre como «experiencia de vida» cargada de una riqueza mayor que la mera experiencia representativa. Efectuar esta distinción le permitió comprender a la vida humana a partir de su historicidad, como un fluir fáctico de conexiones encaminadas a la consecución de fines. El elemento de las ciencias del espíritu es el mundo humano vivido, captado en la percepción interna, en el contacto con la vida. Así se entiende por mundo humano al conjunto de singularidades, vivencias y manifestaciones objetivadas de vida que lo integra, hecho que justifica la propuesta de un dualismo metodológico. 


\section{ALGUNAS CONSIDERACIONES SOBRE LA NOCIÓN DE ESPÍRITU}

Dilthey postula su noción de vida en clara confrontación con la concepción racionalista de la Modernidad. Taylor recuerda que esta Época, una vez desprovista del orden aplicado por la visión teológica buscó un nuevo principio regulador del mundo. Surge así la razón como el criterio que todo lo ordena, pero la novedad está en que a él se accedería a partir de la observación y el pensar. El poder de conocimiento de la razón humana, su fuerza para obtener conocimientos válidos, condujo a la gestación de corrientes filosóficas de tipo naturalista, mecanicistas, atomistas y homogenizadoras. La cosmovisión racional, que propicia la noción moderna del sujeto autónomo, viene acompañada con la consecuente idea de su poder y dominio sobre el mundo. En Alemania, este proyecto racionalista se articulo con el pensamiento religioso. Las visiones cristianas reformistas del luteranismo y calvinismo tomaron esta idea del dominio del hombre sobre la tierra pero considerando que él a su vez se subordinaba a un poder mayor y creador (Dios). Esta idea influyó en la cosmovisión de la filosofía alemana del siglo XVIII que intentó postular una visión desacralizada de la misma [cf., Taylor 1999, pp. 3-14]. El objetivo de esta generación era devolverle al hombre su unidad con la naturaleza pero dejando intactos sus formas vitales de autonomía (conciencia, moral y libertad), para lo cual desarrollan la noción de espíritu. Con ella buscaban expresar ese plano dinámico del mundo humano distinto a la estructura «estática» de la naturaleza aunque relacionados y no simplemente opuestos [cf., Ibid., pp. 38-40]. En este contexto toman fuerzas las ideas del romanticismo. Hegel, como parte de esta generación, absorbió esta corriente romántica tal como se puede apreciar en la utilización del término "espíritu». No obstante, deja de lado el elemento irracional del romanticismo en pos de una constitución racionalista, especialmente en sus obras de madurez.

En términos generales se asocia la noción de espíritu con la de nous griego para señalar una realidad que trasciende la vida orgánica (mera vida natural) [Cf., Inwood 1992, p. 274]. Como se expuso, dicho término es retomado por el idealismo alemán para contraponer una realidad dinámica (intelecto o pensamiento) distinta a la naturaleza «estática». Inwood expone que en esa época en Alemania el término «Geist» refiera a la profundidad (Profundity) y la amplitud (Breadth). Así se puede entender el término «Geist» por la vivacidad y actividad del intelecto o pensamiento que se fenomenaliza en un tiempo y espacio propio, en oposición a la noción de naturaleza (Natur) que se somete a la dinámica espacio-temporal de sus leyes causales. Por su parte, Gadamer considera que la noción de espíritu expresa un espacio de vida, una 
temporalidad cotidiana que articula pasado, presente y futuro [Gadamer 2003, p. 42]. Así el espíritu apela a un horizonte temporal heredado y un futuro abierto, concepción que, como se verá, se asemeja al uso que de dicho término hace Dilthey; su noción de espíritu parte del diálogo que él entabla con el complejo mundo filosófico del cual hereda el término; un recorrido por el antecedente inmediato por él reconocido posibilitará apreciar sus diferencias y la novedad de su propuesta.

Hegel es el espejo del cual Dilthey toma la noción de espíritu para luego darle su propia interpretación, por tanto, es necesario volver a él para comprender la propuesta diltheyana..$^{5}$ Inwood sostiene que en un sentido general el término «Geist» en Hegel se refiere a la actividad humana y sus producciones en contraste con la Naturaleza y la Idea Lógica [cf. Ibid., p. 275]. No obstante, el concepto de «Geist» en Hegel es problemático y multifacético. Por su parte Lu De Vos muestra que entre las muchas acepciones de la noción de «espíritu» en Hegel se puede apreciar que, por ejemplo, los diversos «espíritus del pueblo» que conforman el «mundo espiritual» en tanto formas particulares del «espíritu universal». La realidad espiritual en toda su extensión es el momento de la elaboración de una duración determinada en la «historia del mundo» donde se muestra la «actividad del espíritu» [cf., de Vos 2006, p. 225].

Como se dijo Dilthey toma su noción de espíritu en clara alusión a Hegel, en este sentido, para comprender su apropiación de dicho concepto es relevante exponer aquellos elementos de la obra de Hegel que pueden avalar la lectura que Dilthey hace de su filosofía. Se puede presentar brevemente la posición de Dilthey respecto de Hegel diciendo que entre la historia y la razón (hecho que marca una estructura aporética más no una contradicción) Hegel se decide por la razón en tanto Dilthey lo hace por la historia. En este sentido la interpretación de Dilthey de un espíritu asociado a la vida se sostiene sobre una crítica a la lógica racional del «espíritu» llevada a cabo por Hegel. ${ }^{6}$ Esto se puede apreciar en 1910 cuando Dilthey al precisar su noción de «espíritu» la equiparar, aunque con salvedades, a la de «espíritu objetivo» de Hegel [cf., Dilthey 1944a, p. 173 (GS VII, 149)]. Para sorpresa del lector Dilthey no cita aquí a la Enciclopedia de las Ciencias Filosóficas, donde la división es

5 Como se expuso ut supra Dilthey toma la expresión «ciencias del espíritu» de Mills, no obstante, el término «espíritu» es herencia de los desarrollos de Hegel y la disputa que él mantiene con las implicancia del idealismo hegeliano.

6 La interpretación diltheyana de la obra de Hegel se enmarca dentro de la lectura neokantiana propia de su época. Esta interpretación hoy se ha dejado de lado. Como un ejemplo de las interpretaciones actuales de la filosofía de Hegel en relación al espíritu Lu de Vos sostiene que dicho término no es el concepto central en Hegel, pues él es solo una parte de la realización de la Idea, complemento opuesto a la naturaleza [cf., de Vos, Ibid., pp. 222-3]. 
explicitada por Hegel, sino a la Fenomenología del Espíritu.

En la Enciclopedia de las Ciencias Filosóficas, Hegel presenta la división del espíritu según su desenvolvimiento [cf. de Vos, Ibid, pp. 224-6]. Allí, sostiene que el espíritu subjetivo consiste en el momento donde el espíritu se encierra en sí mismo y no se realiza más que para él. El espíritu objetivo, por otra parte, consiste en la salida del espíritu fuera de sí. Es el momento en que el espíritu forma, como su producto, la realidad del mundo. El espíritu objetivo es la historia de un pueblo, una sociedad o una época; encierra en sí las costumbres, los usos, las leyes, la moral y la política. Cabe aclarar que no es la mera suma de conciencias individuales, él posee realidad y vida propia, realizándose a través de los individuos a los cuales forma al imprimirles su sello. Por ello, el espíritu objetivo es la actividad del espíritu que da sentido a la historia, siendo el hombre solo su instrumento para tal fin. Dilthey también ve aparecer en las Lecciones sobre Filosofía de la Historia de Hegel esta concepción del hombre como momento de la Idea, como un instrumento de la Lógica de la Razón que se manifiesta en la historia. En dicha obra el individuo pasa a ser un momento en el proceso de realización de lo universal. [Cf. Hegel 2005, p. 29 (Werke XII, pp. 39-40) $]^{7}$ Dilthey entienden que para Hegel el individuo se encuentra subsumido a la Idea, como un momento de su devenir en búsqueda de su libertad y autoconciencia. Finalmente, en la Enciclopedia el Espíritu Absoluto es la verdad absoluta del espíritu, el en sí y el para sí. Espíritu subjetivo y objetivo son la Idea Absoluta que no existe aun de modo acabada. Estas concepciones hegelianas fueron interpretadas por Dilthey como una visión excesivamente racional y universalista del mundo que posee estructuras metafísicas intrínsecas por lo cual no puede ser considerada una posición lo suficientemente crítica, ni ser la base que sustente una fundamentación para las ciencias del espíritu .

7 A tales efectos véase el tratamiento de la figura de Julio Cesar por parte de Hegel como ejemplo del papel de los grandes hombres en la historia.

Aquí se toma la edición de Suhrkamp pues corresponde a la versión de Karl Hegel en 1840, única a la que Dilthey pudo haber tenido acceso. En este sentido se deja de lado la recopilación efectuada por Lasson-Hoffmeister y editada por Meiner, ya que la misma fue publicada luego de la muerte de Dilthey. De esta última se posee una traducción al castellano traducida por Ortega y Gasset y publicada por la editorial Revista de Occidente; la de 1840 fue traducida por Suda bajo la editorial Claridad.

8 Dilthey define a la metafísica como una interpretación intelectualista del mundo [Dilthey 1978b, p. 61. (GS VIII, p. 68)]. Según señala Bambach, Dilthey entiende a la metafísica en el sentido aristotélico de filosofía primera. Para dicho intérprete, Dilthey concibe que el principio rector de la metafísica es el «principio de razón suficiente», fundamento de todos los fenómenos mundanos. No obstante, sostiene que ella como investigación de los primeros principios es puramente lógica y abstracta, la cual debe ser superada por una indagación científico-empírica; hecho que no conduce a la desaparición de la metafísica, sino un claro límite a su campo de 
Como se expuso, al intentar precisar su noción de «espíritu» Dilthey recurre a la Fenomenología del Espíritu. Según su interpretación, en dicha obra culmina el camino filosófico del «joven» Hegel, aquel al cual él reconocía por su noción de vida y fraternidad. Para Dilthey, en la Fenomenología del Espíritu aun no se expresa la rigidez del sistema idealista del Hegel «maduro» que, según su interpretación, obtura el movimiento histórico al imponer una estructura lógica idealista. No obstante, dentro de la lectura diltheyana, el espíritu hegeliano siempre conserva la idea de un universal que se despliega a sí mismo en búsqueda de su autoconciencia, consagrada en la noción de espíritu absoluto. ${ }^{9}$ Para Dilthey, en Hegel lo racional discurre en el devenir de una Idea, ahora Espíritu Absoluto, la cual para lograr la autoconciencia requiere salir fuera de sí. La realidad se expresa a sí misma como el proceso de las determinaciones de la razón; según su lectura esta visión idealista también se traslada a la historia.

Dilthey rescata que en la Fenomenología del Espíritu Hegel muestra la penetración histórica del espíritu a través de distintos momentos. En la historización de la conciencia Hegel encuentra la ley del movimiento dialéctico y la concepción del hombre como un ser histórico [Dilthey 1944b, p. 171 (GS IV, p. 157)]. Cuando Dilthey, citando la segunda sección de la Fenomenología del espiritu, sostiene que lo que él concibe como espíritu es equivalente al espíritu objetivo de Hegel también decide marcar su distanciamiento [Dilthey 1944a, p. 172 (GS VII, p. 148)]. La siguiente cita muestra claramente la diferencia:

Entiendo por tal espíritu objetivo las formas diversas en las que la comunidad

acción [Cf., Bambach 1995, p. 136]. Asimismo, recuérdese que para Dilthey la empiria no implica un empirismo crudo de tipo positivista (al que Dilthey califica igualmente de metafísico) sino el plano del mundo histórico donde acontecen las «experiencias de la vida». Él entiende que el abordaje empírico del mundo humano no puede darse de modo directo (contemplación pura), puesto que siempre se encuentra mediado por formas culturales-científicas-históricas; hecho que hace necesaria la reflexión filosófica que permita explicitarlas.

9 Podría calificarse como «panteísta» a la interpretación diltheyana de las obras del Hegel «maduro». Si bien Hegel busca clarificar la teología cristiana, su noción de Dios-Espíritu no es equiparable con la del teísmo tradicional. Para Hegel el espíritu (Dios) no puede existir independientemente de los hombres. Por el contrario, es un espíritu que vive como tal solo a través de los hombres. Ellos son los vehículos indispensables de su existencia. Pero al mismo tiempo, Geist no se puede reducir al hombre, no es idéntico con el espíritu humano, ya que es también la realidad espiritual infinita de lo universal como un todo [cf., Taylor, op. cit., pp. 445]. La noción de espíritu infinito, en opinión de Taylor, también debe ser asociada con la idea hegeliana de destino [cf., Ibid., p. 79]. Según dicho intérprete, esta constitución idealista del espíritu hegeliano favoreció las acusaciones de panteísmo o espinosismo aunque, como también dicho autor señala, las mismas se sustenta sobre errores interpretativos [cf., Ibid., p. 87]. 
que existe entre los individuos se ha objetivado en el mundo sensible. En este espíritu el pasado es para nosotros presente permanente. Su ámbito alcanzan desde el estilo de vida, desde las formas de trato hasta la conexión de fines que la sociedad se ha establecido, las costumbres, el derecho, el estado, la religión, el arte, las ciencias y la filosofía. [Ibid., p. 232 (GS VII, p. 208)].

Dilthey concibe su noción de espíritu objetivo incorporándole el arte, la religión y la filosofía, es decir, el espíritu absoluto de Hegel. Esta readaptación se encuentra justificada por sus críticas (expuestas ut supra) al Hegel «maduro» y su «sistema». Según Dilthey el espíritu objetivo es el resultado de la acción humana, del curso vital donde lo dado establece conexiones históricas. En tanto exteriorización y objetivación histórica el espíritu es el resultante de las conexiones dadas en el «curso vital». En este sentido se puede adelantar ahora que el espíritu es para Dilthey un ser históricamente estructurado a partir del cúmulo de manifestaciones, expresiones y recuerdos dados por la acción humana. Es decir, el espíritu es solo el resultado de las interacciones del hombre en tanto ser histórico y activo.

\section{LA NOCIÓN DE ESPÍRITU EN DILTHEY}

De lo expuesto en el punto anterior se desprende que Dilthey privilegia un tipo de noción de espíritu por sobre otras. Deja de lado la idea de un «espíritu subjetivo», aquel que psicológicamente se encierra en la mera actividad de una conciencia; no obstante, como se verá, retoma al individuo como agente histórico a partir de su noción de «hombre como punto de cruce». También elimina cualquier apelación al «Espíritu Absoluto», aquella noción del sistema hegeliano que hace del Espíritu una manifestación de la Idea o de una estructura lógica supra-histórica. Al equiparar su noción de espíritu con la de «espíritu objetivo» Dilthey recupera el carácter histórico propio de las acciones humanas. El espíritu es el resultante del contacto dado entre múltiples conexiones históricas, el producto del curso vital creador de estructuras duraderas que anteceden y perduran a la vida individual.

Si bien es cierto que Dilthey toma la noción de ciencias del espíritu en contraposición a las ciencias naturales, definiendo a aquella como el conjunto de ciencias particulares entre las cuales se pueden enumerar la psicología, la antropología, las ciencias morales, la historia y la filosofía entre otras [Dilthey 1944a, p.100 (GS VII, pp. 79-80)]; no es menos cierto que el hecho primordial no es la delimitación de ámbitos científicos sino la puesta en valor de la vida histórica, la acción humana y sus objetivaciones. En línea con el idealismo alemán Dilthey concibe al espíritu en oposición a la naturaleza, siendo ella lo pasivo en tanto aquél refiere a la vida activa. Al reconocer la imprecisión como elemento característico del término «espíritu» Dilthey 
expresa la imposibilidad de cualquier postulación de una naturaleza humana supraterrenal o una naturaleza innata e inmutable del hombre. La expresión «hechos espirituales» refiere al la actividad del hombre en tanto ser histórico, un «punto de cruce», ser cuyas acciones están en constante relaciones con el curso vital y las distintas objetivaciones. En otros términos, el espíritu es un ser histórico, consiste en el cúmulo de manifestaciones, expresiones y recuerdos del mundo humano [cf., Ibid., p. 303 (GS VII, p. 277)].

El descubrimiento de la historicidad de la vida humana le impuso la necesidad de repensar la noción moderna de experiencia. Dilthey entiende que ella es una forma de captación dominada por el pensamiento conceptualizador que busca conocer; no obstante, el dominio del par experiencia-conocimiento es el resultado de un recorte de las múltiples formas de «experiencias de vida», hecho que requiere de una noción más abarcativa y propia del mundo humano; hacia este último apuntan su par vivencia-comprensión. En otras palabras, la diversidad del mundo humano está dada por las características del hombre entero (aquel que siente, quiere y el piensa). La experiencia es un recorte de la vida humana pues reduce su actividad solo a la acción del pensamiento generador de abstracciones, hipótesis axiomáticas y leyes naturales (causalidad). Aquí experiencia es solo acción del pensar que, en pos de conocer, deja de lado el sentir y el querer y conduce a la subordinación de los hechos espirituales (la acción humana) dentro de los naturales [ $c f$., Dilthey 1949, pp. 19-22 (GS, I, pp. 11-14)].

En relación con el término vivencia Gadamer en Verdad y Método expone un recuento histórico de su aparición en la lengua alemana y remarca que este término, de origen romántico, busca recuperar, frente al domino del pensar racional, el sentir y la interioridad que cada individuo experimenta [Gadamer 2012, pp. 96ss. (WM I, p. 66)]. Jay diferencia correctamente la noción de experiencia manejada por las corrientes empiristas y las afines a ella como las kantianas (que la reducen a un cúmulo de percepciones sensibles) de la concepción diltheyana de vivencia. Esta última no es, como bien sostiene Jay, el mero encierro en el sujeto (un solipsismo que retira al hombre del mundo), sino más bien el modo en que el hombre está en el mundo. ${ }^{10}$ Para Dilthey la vivencia es una forma originaria de estar en el mundo, es el modo que el hombre, en tanto ser vivo, capta el mundo de modo inmediato y sin abstracciones del pensar. ${ }^{11}$ Esta vivencia es la «célula original» (Urzelle) del mundo histórico-

10 No obstante, dicho intérprete traduce revivencia (Nacherleben) como re-experiencia (re-experiencing) lo que requiere, para evitar yerros interpretativos, algunas aclaraciones (las cuales dicho autor no realiza), pues sin ellas se podría interpretar erróneamente que Dilthey vuelve a introducir a la vivencia dentro de la noción de experiencia propia de las ciencias naturales. [Cf. Jay 2009, p. 270].

11 En relación con las objeciones dadas al término vivencia por su carga inmediata y 
humano y el dato básico de toda ciencia del espíritu [cf., Dilthey 1944a., p. 185 (GS VII, p. 215)]; del cual el hombre tiene un «conocimiento tácito» que se manifiesta históricamente en características cualitativas, patrones múltiples, significados, valores y relaciones vivenciales, formas objetivadas de la cultura y las relaciones intersubjetivas. Moya Espí postula dos modos de interpretar a la vivencia en Dilthey, una como percatación (contacto inmediato con la vida) y otra como significativa (el conjunto de signos y símbolos que socialmente se constituyen a lo largo de la vida y la historia) [Moya Espi 1981, pp. 247ss.].

Este contacto inmediato con la vida abre al hombre al mundo para construir sus mediaciones históricas. La vivencia en términos simbólicos es el resultado de la actividad humana histórica. Así esta vivencia expresa en términos de contacto con la vida lo mismo que la noción de espíritu objetivo; son formas en que el hombre interactúa con su mundo. Cuando Dilthey concibe al hombre como un ser «entretejido», un «punto de cruce» con el curso vital, sostiene que sus vivencias (incluso su estructura psíquica) son el fruto del contacto con el «espíritu objetivo», la «comunidad» y las distintas «generaciones.» [cf. Dilthey 1944a, p. 304 (GS VII, p. 278) / 1949, pp. 45-6 (GS I, p. 37)].

En conclusión se puede sostener luego del recorrido transitado que para Dilthey el hombre es el resultado del contacto con las formas culturales e históricas que le antecedieron, aquellas con las que se relaciona (vivencialmente) en su presente y las que perdurarán más allá de su tiempo histórico particular. El «espíritu objetivo» es ese mundo de significados dados

su cerrazón en la conciencia es necesario recordar que para Dilthey todo lo captado debe ser dado a la conciencia, pero no todo es meramente un acto de la conciencia. Él se separa de lo que denomina como fenomenismo, la corriente filosófica que sostiene que todo lo captado es una representación de la conciencia. Él define a lo dado a la conciencia como «vivencia» pues expresa el contacto directo (sin mediaciones representativas de la conciencia intencional) con la vida. Dilthey postula un principio basado en la totalidad de las experiencias sustentadas sobre la «conexión de la vida». Los hechos de la conciencia son para Dilthey el conjunto de experiencias performativas, interactivas y reflexivas brindadas por la «conexión de la vida». De este modo busca evitar las tendencias ahistóricas (filosofía de la conciencia y teoría del conocimiento) en pos de adentrarse en la vida humana (psicología descriptiva y hermenéutica). Así Bambach sostiene que los estadios de la conciencia exponen a la vivencia como el modo concreto en que se manifiesta el actuar humano en sus tres planos, pensar, sentir y querer [cf. Bambach op. cit., pp. 153ss.]. Sin embargo, no se puede postular como él lo hace que la concepción diltheyana en torno al principio de fenomenalidad acarree la perspectiva cartesiana [cf. Ibid, p. 183]. Dilthey pone a la conciencia en el tiempo histórico, no subordina el tiempo a las condiciones de la conciencia. Tampoco presenta su teoría de la autognosis en términos cartesianos, ni concibe al método de las ciencias del espíritu como una prolongación epistemológica del de las ciencias naturales. Si se puede sostener que la disputa epistemológica con el modelo naturalista de la experiencia llevó la discusión al plano de la objetividad y la universalidad generando el problema de la relatividad histórica asociado a la vivencia. 
como resultante de las distintas articulaciones del «curso vital» y la vivencia es el modo en que el individuo capta su vida dentro de las conexiones que de dicho curso objetivo. ${ }^{12}$ La vivencia no es mera percatación, interioridad, también requiere de una trama que de cuenta de la pertenencia a la vida, de su participación con el curso fáctico que envuelve holísticamente al mundo humano y lo dota de sentidos y significados. El conjunto de exteriorizaciones significativas de la vida de los hombres es el espíritu, sobre cuyas bases es posible la comprensión. En otras palabras, son las estructuras significativas de la historicidad de la vida humana, estructuras comprensivas, las que hace posible la comprensión histórica (en tanto interpretación técnica). La vida como curso es plenitud en el tiempo presente, pertenencia al pasado en el recuerdo y expectativa en referencia al futuro.

Y la 'presencia' consiste en el resultar incluido lo pasado en nuestra vivencia; aquello que de esta suerte constituye en el corriente del tiempo una unidad vivencial porque tiene un significado unitario en el curso de la vida compone la unidad más pequeña que podemos designar como vivencia. Pero por encima de esto, el uso del lenguaje designa también como vivencia a toda unidad, ideal, más amplia de partes de vida que tiene un significado en el curso de la vida y emplea también este concepto en el caso en que los momentos se hallan separados por acontecimientos interruptores. [Dilthey 1944a, p. 94 (GS VII, p. 72)]

La condición histórica del hombre en tanto ser comprensivo tiene fundamentalmente que ver con la experiencia primaria de sentirse parte de un curso vital significativo, de un devenir generacional. El concepto de espíritu es el modo en que Dilthey entiende se puede expresar este vasto y complejo modo de interacción y articulación que tiene el mundo humano y sus formas histórico-culturales objetivadas. El espíritu no es nous, un intelecto, sino vida resultante de la acción histórica del hombre en tanto ser que piensa, siente y quiere.

\section{REFERENCIAS BIBLIOGRÁFICAS}

BAMBACH, C. 1995: Heidegger, Dilthey and the Crisis of Historicism. Ithaca: Cornell University press.

DE VOS, L. 2006: «Geist» en: Cobben, P. y De Vos, L., (Editores), Hegel Lexikon. Darmstadt: Wissenschaftl Buchgesell.

DILTHEY, W. 1944a: El Mundo Histórico, tr. Imaz. México: FCE.

DILTHEY, W. 1944b: Hegel y el idealismo, tr.. Imaz. México: FCE.

12 El significado se expresa: «Precisamente en las relaciones entre este presente, el pasado y el futuro consiste el carácter del curso de nuestra vida.» [Cf. Dilthey 1944a, p. 94 (GS VII, p. 73)] 
DILTHEY, W. 1945: De Leibniz a Goehte, tr. Imaz, y otros. México: FCE.

DILTHEY, W. 1949: Introducción a las Ciencias del Espíritu, tr. Imaz.México: FCE.

DILTHEY, W. 1978a: Psicología y Teoría del Conocimiento, tr. Imaz. México: FCE.

DILTHEY W. 1978b: Teoría de las Concepciones del Mundo, Trad. Imaz, México, FCE.

DILTHEY, W., (GS I), Gesammelte Schriften, Band I, Einleitung in die Geisteswissenschaften. Stuttgart-Göttingen: B. G. Teubner/ Vandenhoeck \& Ruprecht, 2008.

DILTHEY, W., (GS IV), Gesammelte Schriften, Band IV, Die Jugendgeschichte Hegels und andere Abhandlungen zur Geschichte des Deutschen Idealismus, Stuttgart-Göttingen: B. G. Teubner/ Vandenhoeck \& Ruprecht, 1990.

DILTHEY, W., (GS V), Gesammelte Schriften, Band V, Die geistige Welt. StuttgartGöttingen: B. G. Teubner/ Vandenhoeck \& Ruprecht, 1990.

DILTHEY, W., (GS VII), Gesammelte Schriften, Band VII, Der Aufbau der gesichtlichen Welt in den Geisteswissenschaften, 1992.

DILTHEY, W., (GS VIII), Gesammelte Schriften, Band VIII, Weltanschauungslehre. Stuttgart-Göttingen: B. G. Teubner/ Vandenhoeck \& Ruprecht, 1991.

GADAMER, H-G. 2003: La actualidad de lo bello, tr. Gomez Ramos, Buenos Aires, Paidos.

GADAMER, H-G. 2012: Verdad y método, tr. Aparicio-Agapinto, Tomo I. Salamanca: Sígueme.

GADAMER, H-G., (WM I), Gesammelte Werke, Band I, Wahrheit und Methode, Tübinga: J.C.B. Mohr, 1990.

HEGEL, G. W. F. 2005: Filosofía de la Historia, tr. Suda. Buenos Aires: Editorial Claridad.

HEGEL, G. W. F. 2006: Fenomenología del Espíritu, trad. Roces. México: FCE.

HEGEL, G. W. F.: Werk III, Phänomenologie des Geistes. Frankfurt am Main: Suhrkamp, 1989.

HEGEL, G. W. F.: Werke XII, Vorlesung über die Philosophie der Geschichte. Frankfurt am Main: Suhrkamp, 1989.

INWOOD, M. 1992: A Hegel Dictionary. Massachusetts: Wiley-Blackwell.

JAY, M. 2009: Cantos de Experiencia. Variaciones Modernas sobre un Tema Universal, Buenos Aires: Paidos.

MAKKREEL, R. 2010, «Wilhelm Dilthey and the Neo-kantians: on the conceptual distinctions between Gesteswissenschaften and Kulturwissenschaften», en: Makkreel, R. y Luft., S. (eds.), Neo-Kantianism in Contemporany Philosophy, Indiana: Indiana University Press.

MAKKREEL, R. 1992: Dilthey, Philosopher of the Human Studies, New Jersey: Princeton University Press.

MOYA ESPI, C. 1981: Interacción Histórico-Social y Subjetividad en la Obra de 
Wilhelm Dilthey. Valencia: Universidad de Valencia, (Tesis doctoral).

RODI, F. 2003: Das struckturierte Ganze. Studien zum Werk von Wilhelm Dilthey. Weilerswist: Velbrück Wissenschaft.

RITTER, J. 1974: Historisches Wörterbuch der Philosophie, Band 3. Basel: Schwabe \& Co.

TAYLOR, C. 1999: Hegel. New York: Cambridge Press.

Luis María Lorenzo es Becario posdoctoral del Consejo Nacional de Investigaciones Científicas y Técnicas en la Universidad Nacional de La Plata

Líneas de Investigación:

Filosofía de la Historia y Hermenéutica

Publicaciones recientes:

(2016): “Consideraciones en torno a las aporías en Wilhelm Dilthey", Eidos 25: 14 42, ISSN 2011-7477.

(2014): "Narración y construcción histórica en Walter Benjamin", El arco y la Lira. Tensiones y Debates 2: 79-91, ISSN 2344-9292.

Correo electrónico: luism.lorenzo@gmail.com 